\title{
Physics-aspects of dose accuracy in high dose rate (HDR) brachytherapy: source dosimetry, treatment planning, equipment performance and in vivo verification techniques
}

\author{
Antony Palmer, MSc', David Bradley, PhD', Andrew Nisbet, PhD'.3 \\ IDepartment of Physics, Faculty of Engineering and Physical Science, University of Surrey, United Kingdom, 2Medical Physics Department, \\ Queen Alexandra Hospital, Portsmouth Hospitals NHS Trust, Portsmouth, United Kingdom, 3Medical Physics Department, Royal Surrey County \\ Hospital NHS Foundation Trust, Guildford, United Kingdom
}

\begin{abstract}
This study provides a review of recent publications on the physics-aspects of dosimetric accuracy in high dose rate (HDR) brachytherapy. The discussion of accuracy is primarily concerned with uncertainties, but methods to improve dose conformation to the prescribed intended dose distribution are also noted. The main aim of the paper is to review current practical techniques and methods employed for HDR brachytherapy dosimetry. This includes work on the determination of dose rate fields around brachytherapy sources, the capability of treatment planning systems, the performance of treatment units and methods to verify dose delivery. This work highlights the determinants of accuracy in HDR dosimetry and treatment delivery and presents a selection of papers, focusing on articles from the last five years, to reflect active areas of research and development. Apart from Monte Carlo modelling of source dosimetry, there is no clear consensus on the optimum techniques to be used to assure dosimetric accuracy through all the processes involved in HDR brachytherapy treatment. With the exception of the ESTRO mailed dosimetry service, there is little dosimetric audit activity reported in the literature, when compared with external beam radiotherapy verification.
\end{abstract}

Key words: accuracy, brachytherapy, dosimetry, high dose rate (HDR), in vivo, treatment planning.

\section{Purpose}

The need for dosimetric accuracy in external beam radiotherapy is well established [1,2]. A standard requirement of the combined uncertainties in absorbed dose delivery is often stated as 3.5\% at one standard deviation, first quoted by Mijnheer [3]. Dosimetric accuracy of high dose rate (HDR) brachytherapy may also be expected to contribute to the achievement of clinical treatment aims, tumour control and minimised normal tissue toxicity, due to the steepness of the clinical dose effect curves. Van Dyk et al. [4] defined a requirement for brachytherapy treatment delivery of $3 \%$ accuracy in dose at distances of $0.5 \mathrm{~cm}$ or more at any point for any radiation source. Clinical trials also depend upon dosimetric accuracy and consistency between participating centres. The dosimetric challenge is particularly difficult in brachytherapy due to small treatment distances, very high dose gradients and orders of magnitude variation in dose deposition across volumes of interest. It may also be reasonably claimed that dosimetric accuracy is also required to avoid treatment errors: IAEA Safety Re- port Series No 17 [5] "Lessons learned from accidental exposures in radiotherapy" discussed 32 incidents involving brachytherapy. ICRP 86 "Prevention of accidental exposures to patients undergoing radiation therapy" [6] noted potential errors in brachytherapy arising from problems with equipment, calibration, treatment planning, and treatment delivery. ICRP 97 "Prevention of high-dose-rate brachytherapy accidents" [7] reported more than 500 accidents involving HDR brachytherapy. The field of brachytherapy physics, equipment and clinical techniques is undergoing significant innovation and rapid modernisation, including enhanced use of imaging, improved dosimetry and treatment planning, and patient-specific optimisation [8,9]. It is of course essential that the requirements for dose accuracy are considered and met in all of these developments.

The scope of 'physics-aspects of dose accuracy' reviewed in this paper is illustrated in Fig. 1 and includes knowledge of the dose field around the source, creation of the treatment plan, performance of equipment and verification of dose delivery. Particular emphasis is placed on reviewing 
 process}

HDR source: dose rate distribution

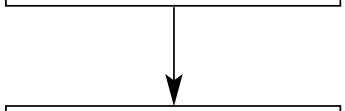

Clinical dose calculation: treatment planning system
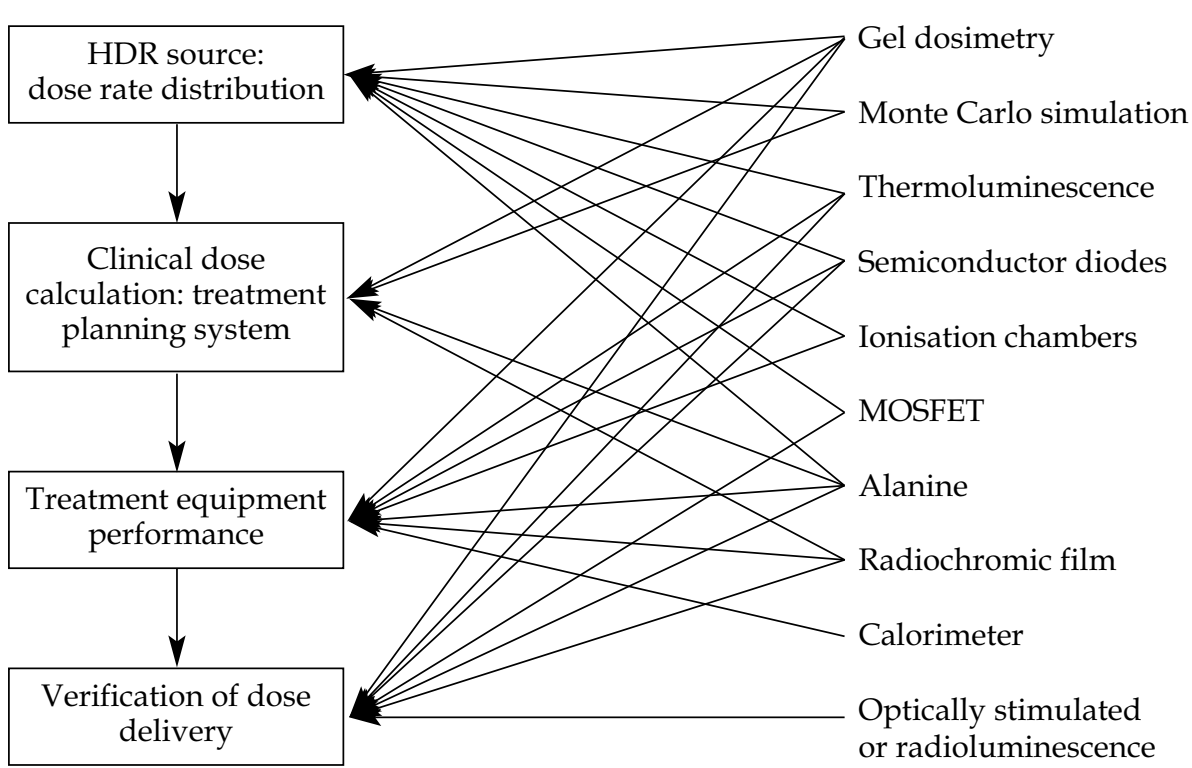

Tools applied

Fig. 1. HDR brachytherapy processes, considered in this review, that influence dosimetric accuracy, with a selection of tools reported in the literature that have been applied to assess dosimetric accuracy

the range of measurement techniques available that have been applied to HDR brachytherapy. We do not include the important uncertainties arising outside the brachytherapy clinic, such as those at the accredited dosimetry calibration laboratories [10]. The reader is also directed to the comprehensive review by DeWerd et al. [11] which addresses uncertainties of single-source dosimetry in brachytherapy preceding clinical use. Accuracy is defined as the level of agreement of a measured parameter to its true value, also the degree of correctness of a quantity. In the context of this work, accuracy between the dose prescribed by the clinician and that delivered in a patient is considered. The main contribution is a range of uncertainties, from source dosimetry to planning algorithm performance. However, one may also consider how well the dose distribution can be optimised to match the clinically intended dose distribution, in terms of dose delivery accuracy.

Of course 'physics-aspects' are not the only contributors to dose delivery uncertainty in brachytherapy and indeed the more 'clinically' related aspects may have more, or even overwhelming, influence on the treatment outcome than the 'physics' aspects discussed here. The clinical consideration arise from a multitude of sources [12] including clinical target volume definition and contouring, user operation of equipment, human factors in applicator positioning, internal organ motion and inter- and intra-fraction applicator movement $[13,14]$. The imaging resource and technique employed may also have a dramatic influence on overall uncertainties in dose delivery, ranging from 2D orthogonal imaging to MRI/CT fustion $[15,16]$. All of the physicsprocesses illustrated in Fig. 1 should be considered in terms of dosimetric accuracy and uncertainty to ensure the highest quality brachytherapy treatment delivery. A number of tools have been developed in recent years and are active- ly being refined to address dosimetry requirements, which may have application in multiple ways, as shown in Fig. 1, from establishing fundamental source dosimetry in terms of absolute dosimetry traceable to a primary standard and dose distribution around a source to in vivo verification of treatment delivery.

\section{Material and methods}

A systematic review was conducted of journal articles discussing concepts, research, and practices of dosimetry in HDR brachytherapy published from 2002 to 2012. Medline and Embase databases were searched with keywords "high dose rate", "brachytherapy", "dosimetry" and alternative forms, limited to English language. 116 articles were found of which 84 were directly relevant for the purposes of this review. This information was supplemented by reviewing reference lists and personal files.

\section{Review of physics-processes in HDR brachytherapy}

\section{Source dosimetry}

A number of experimental methods have been used to measure the dose distribution and anisotropy functions of HDR sources. These include thermoluminescent dosimeters [17-20], ionisation chambers [21], semiconductor diodes [18], MOSFETS [22,23], radiochromic film [24-28] and Polymer or Fricke gel dosimetry [29]. Theoretical Monte Carlo (MC) calculations have also been extensively utilised $[17,18$, 30-35]. MC simulation [36] has now become the gold-standard accepted method for determination of dose deposition parameters (dose rate distribution) around a brachythera- 
py source, expressed using parameters as recommended in the AAPM Task Group No. 43 Report (TG-43 and TG-43 U1) $[37,38]$ : the dose rate constant $(\wedge)$, the geometry factor $G(r, \theta)$, the radial dose function $\mathrm{g}(\mathrm{r})$, and the $2 \mathrm{D}$ anisotropy function $\mathrm{F}(\mathrm{r}, \theta)$. The AAPM report recommends that these factors can be obtained by experiment or by MC simulation, with the AAPM Brachytherapy Subcommittee carefully supervising the process and acceptance of data in relation to defined prerequisites [39]. As early as MC methods were employed to evaluate the dosimetry of HDR brachytherapy sources [40].

Validation of the MC calculated dose deposition parameters for specific HDR sources has received much attention. Chandola et al. [41] have compared results of MC codes, EGSnrc and GEANT4, alongside validation by ionisation chamber measurements, for the Nucletron mHDRv2 Ir-192 brachytherapy source, finding the two codes gave comparable results in TG-43 dosimetric data: agreement of dose rate constant within $1 \%$, radial dose function within $2 \%$ up to $5 \mathrm{~cm}$ and within $6 \%$ up to $10 \mathrm{~cm}$ and $2 \mathrm{D}$ anisotropy function within $2 \%$ for polar angles $25^{\circ}<\theta<140^{\circ}$, up to $6 \%$ outside this range. MC has also been used for evaluation of clinically relevant parameters, rather than simply dose distribution around an isolated HDR source, such as Sureka et al. [33] who calculated relative dose distribution and effective transmission around shielded vaginal cylinders for an Ir-192 source, using MCNP4b MC code. Lliso et al. [42] have successfully used GEANT4 MC code to determine the backscatter and electron contamination from internal shielding in HDR skin applicators. The MC results were verified with radiochromic EBT films at several depths in a phantom, finding agreement within measurement uncertainty.

The quality of radiochromic film has improved over the last decade, with the active layer being more uniform and the film being less sensitive to ambient light. A number of recent studies have successfully used radiochromic film for evaluation of dose distribution around HDR sources [26]. Uniyal et al. [43] have used Gafchromic EBT2 radiochromic film and thermoluminescent dosimeters (TLD) to assess the dose rate constant and radial dose function for the Nucletron microSelectron Ir-192 source. Radiochromic film was chosen for its higher resolution compared to other experimental methods and validated against TLD results, finding agreement within 3.9\% for dose rate constant and 2.8\% for radial dose function. Relative to TLD dosimetry, radiochromic film was reported as being less expensive, easier to use and having improved reproducibility. Aldelaijan et al. [24] have shown that radiochromic film may be used in either solid water-equivalent materials or liquid water when performing radiochromic film based dosimetry with HDR brachytherapy sources, extending its usefulness. The use of polymer gel dosimetry to measure dose distribution around HDR brachytherapy sources was initially complicated due to oxygen contamination and other practical issues [44]. However, new rigid materials that are stable during- and post-irradiation with linear response at low energies and insensitive to oxygen are now available [45]. Wai et al. [46] have demonstrated the use of the solid polyurethane radiochromic dosimeter PRESAGE ${ }^{\mathrm{TM}}$ with opti- cal CT readout as a potential method for 3D dosimetry. Evaluation of the dose distribution produced by the microSelectron-HDR Ir-192 source was determined using the dosimeter and found to be in good agreement with MC calculation, especially at polar angles above $20^{\circ}$. The $3 \mathrm{D}$ dosimeter was used to validate anisotropy functions generated with MCNP4c code, with accuracy of order $5 \%$ achieved. However, Wai states that refinements to the dosimetry method used are necessary before the technique can be applied clinically. Guo et al. [47] have also evaluated the use of PRESAGE ${ }^{\mathrm{TM}}$ with optical CT scanning (OCTOPUS ${ }^{\mathrm{TM}}$ ) concluding the system provides a robust, clinically effective and viable high-resolution relative 3D dosimetry system for brachytherapy. Several other investigators have also contributed to the evidence for dosimetric use of the PRESAGE ${ }^{\mathrm{TM}}$ material $[48,49]$.

Carrara et al. [50] used Fricke gel dosimetry to measure the anisotropy function of an Ir-192 brachytherapy source. A tissue equivalent phantom with Fricke gel-layer dosimeters was manufactured and agreement was found within $3 \%$ of the planning system data at short distances with delivered dose around $15 \mathrm{~Gy}$, but deteriorated in more distant lower dose regions.

Antonovic et al. [51] have measured absorbed dose distributions around two Ir-192 sources with a dosimetry system using lithium formate monohydrate $\left(\mathrm{HCO}_{2} \mathrm{Li} \times \mathrm{H}_{2} \mathrm{O}\right)$ as detector material and electron paramagnetic resonance (EPR) spectroscopy for readout. Relative standard uncertainties in the experimentally determined absorbed dose was estimated in the range 1.3 to $1.7 \%$. Although the energy dependence is less than commonly used thermoluminescent lithium fluoride $(\mathrm{LiF})$, the authors conclude the dosimeter size needs to be reduced (from the $4.5 \mathrm{~mm}$ detectors used in their study) for application to single brachytherapy source dosimetry, where dose gradients are greater (closer than $3.0 \mathrm{~cm}$ from a source), in order to keep the volume averaging correction reasonably low.

Issa et al. [52] have established the use of Ge-doped optical fibres as thermoluminescence dosimeters for brachytherapy applications, including comparison with calculations using DOSRZnrc MC code, finding agreement within $3 \%$ for a Ba- 133 source and $1 \%$ for a Co-60 source.

It is important to consider the size and shape of phantoms used for brachytherapy source dosimetry, in both measurement and simulations. Granero et al. [53] have determined the equivalent spherical phantom size to a variety of cylinder and cube sizes for brachytherapy dosimetry with Ir-192 and Cs-137 sources. It is noted that published literature often employs a variety of phantom dimension and hence different scatter conditions.

\section{Treatment planning}

Rivard et al. [54] have comprehensively reviewed recent developments in TPS algorithms and current research directions and the reader is directed to review this publication. New model-based dose calculation algorithms are being introduced that will radically change the complexity and potentially accuracy of brachytherapy treatment planning. In moving from the simplistic TG-43 formalism, the limitations of liquid water dosimetry and dose distributions 
for fixed scattering conditions are being addressed, enabling dose distributions in the presence of heterogeneities and patient scatter conditions to be evaluated with improved accuracy. Advanced calculation tools, radiobiology models and superior image guidance will shortly be available in modern TPS. In a second paper, Rivard et al. [55] considered commissioning techniques and quality assurance recommendations of TPS utilising model-based dose calculation algorithms. It is essential that a cautious approach is taken to adopting radically new algorithms to ensure the historic basis of brachytherapy prescription dosage is not abandoned. A joint AAPM/ESTRO/ABS working party, AAPM Task Group 186 has been established to consider modelbased brachytherapy dosimetry techniques [56].

The majority of currently available, traditional brachytherapy planning systems simply employ a summation of the pre-determined dose distribution around individual source positions based on TG43 formalism. This will lead to some degree of uncertainty in the clinical treatment delivery. Chandola et al. [41] have quoted the possible magnitude of error in conventional treatment planning system dose calculations using analytical methods as \pm 15 to $20 \%$, which may lead to overdose of organs at risk or under dose of target volume. Chavez-Aguilera et al. [57] have provided a more conservative estimate of the potential error, by comparing a conventional treatment planning system with MC code and CT data, providing a result of $8 \%$ underestimate of the absorbed dose by the treatment planning system, due to density and chemical composition variations. Parsai et al. [58] have considered the effect of stainless steel applicator tubing (Fletcher-Suit-Delclos) in cervical and endometrial cancers, comparing conventional treatment planning system dose predictions with that calculated by MCNP MC code, for an Ir-192 source. A reduction of dose to ICRU Point A of at least 3\% was determined when using the planning system, which did not account for the presence of the steel applicator.

Uniyal et al. [59] used Gafchromic EBT2 radiochromic film to assess the effect of rectal air heterogeneity in Ir-192 HDR treatment of carcinoma of the uterine cervix. Experimental measurements using EBT2 film in a rectal phantom, verified by MC simulations, showed $12.8 \%$ dose reduction to the closest rectal surface compared to the treatment planning system, due to lack of back scattering, whereas at the farthest rectal surface it was $24.5 \%$ higher due to no attenuation. Desbiens et al. [60] have also assessed the effect of inhomogeneities including presence of the applicator, air pockets in the CTV and OARs and tissue heterogeneities using Geant $4 \mathrm{MC}$ code, with the CT data from 38 patients undergoing Ir-192 gynaecologic interstitial HDR brachytherapy. Maximum effect on CTV D90 was $8 \%$ and for V100 and $\mathrm{V} 150,4 \%$ and $10 \%$, respectively. Rectum indices reduced by an average of $3 \%$ and urethra and bladder by less than $1 \%$. The authors concluded taking account of heterogeneities has relatively small effects on dose distributions, however excluding air pockets and applicator volumes from the CTV is important. Lakshminarayanan et al. [61] have explored the suitability of using point kernels for brachytherapy dose calculations to take account of heterogeneities in treatment planning and validated these against $\mathrm{MC}$ calcu- lated dose distributions, finding agreement within 5\% in point doses. The authors propose brachytherapy treatment planning with point kernel dose calculation techniques provides clinically required accuracy with rapid calculation compared to full MC evaluations. DeMarco et al. [62] have shown conventional brachytherapy treatment planning system algorithms are especially prone to calculation errors for HDR surface applicator mould treatments with irregular surface lesions. In work comparing TG-43 calculation algorithm with MCNPx MC code, it was shown that the relative size and shape of the bounding medium surrounding an HDR brachytherapy source can have a significant effect (10 to $45 \%$ ) on the calculated dose distribution relative to a reference TG- 43 calculation algorithm. The changed scatter conditions from TG-43 based planning systems to specific patient treatment situations is comprehensively explored by Granero et al. [53], which is completely applicable to the surface applicator situations reported above.

Zourari et al. [63] have proposed a new method for verification of the dosimetric accuracy of these advanced TPS, utilising a virtual phantom/DICOM-protocol based quality control procedure for Ir-192 HDR brachytherapy. A set of virtual DICOM CT image format phantoms were prepared to test homogeneous and inhomogeneous performance as well as contouring, DVH calculation etc., as is common in external beam TPS. Dosimetry calculations of the advanced planning system were compared to MC generated data with good agreement. The authors concluded that the developed test tools provide an appropriately thorough QC of dosimetric performance and other basic features of both conventional and contemporary brachytherapy TPS.

Zourari et al. [64] and Petrokokkinos et al. [65] have also considered the accuracy of a deterministic radiation transport based brachytherapy treatment planning system (Brachyvision v8.8), validating against MCNP MC code and VIP polymer gel with magnetic resonance imaging, for the cases of single sources, multiple source dwell positions and a shielded applicator. TPS and MC dose distributions were in agreement within $2 \%$, except in the penumbra of the shield where TPS calculation errors increased (validated against measurement). In all other regions agreement between TPS and MC code was within $5 \%$ or $2 \mathrm{~mm}$. The authors compared these results to other planning systems which do not account for shielding in applicators where dosimetry errors of 20 to $30 \%$ may be present at the edge of the unshielded segment.

In addition to uncertainties introduced by TG-43 based dosimetry, the accuracy of the final dose delivery in comparison to that intended by the prescribing doctor is also affected by the level of optimisation that can be achieved during the planning process. While this is properly termed improved 'conformality' rather than improved 'accuracy', these issues are mentioned here for completeness. The conventional use of treatment planning systems for HDR brachytherapy planning is a manual iterative modification of dwell times and/or positions until an acceptable isodose distribution is achieved. Use of inverse planning algorithms [66] has become common although there is understandable reluctance to abandon completely traditional 'loading patterns' which have been in use for many years. There have been numerous studies evaluating modified HDR source 
loading patterns to achieve best conformation of isodoses to the clinically identified target volume, hence improving the accuracy of dosimetric delivery in comparison to that intended. One early example is from Brooks et al. [67] in cervical cancer, in which standard plans to Manchester Point A and conformally loaded plans prescribed to the planning target volume were compared using the Baltas conformal index (COIN), finding the optimised plans achieved superior isodose distributions. Siauw et al. [68] have developed a new approach for HDR brachytherapy planning by directly optimizing the dose distribution based on dosimetric criteria, with a fast heuristic. They have demonstrated clinically acceptable performance in prostate HDR cases.

It is also necessary to check the accuracy of the treatment plan prior to delivery in order to ensure absence of inadvertent errors which could affect dosimetric accuracy. Although manual methods are possible, it is preferable to automate this process, as described by Kumar et al. [69] who developed a software tool in $\mathrm{VC}++$ code for plan verification using TG-43 parameters. The verification software and a conventional treatment planning system were found to agree within $3 \%$ in the majority of clinical cases. Astraham [70] has also implemented an independent check programme that runs on personal digital assistants (PDAs), and Carmona et al. [71] a spreadsheet-based independent calculation procedure.

Finally, crucial to the accuracy of treatment planning is the uncertainties that may be introduced by the imageguided techniques that are employed. Cormack [72] has reviewed the quality assurance issues associated with 3D imaging in brachytherapy. While some aspects are clearly clinical, such as image interpretation and target delineation, the uncertainty of applicator/source localisation and implant reconstruction has potential for significant error if not accurately undertaken.

\section{Equipment performance}

It is essential to comprehensively commission brachytherapy treatment units to fully assess their performance characteristics to ensure accurate dose delivery, as reported by Palmer et al. [73]: Autoradiography of source dwell positions, video camera analysis of both source transit profiles and dwell times, corrections for transit doses, as well as absolute dosimetry of the source were all considered in the commissioning. Many techniques have been proposed to measure dose inaccuracies due to transit dose of the moving HDR source to the intended dwell position. Sahoo [74] proposed a well-chamber measurement technique and Calcina et al. [75] have developed a novel method using alanineEPR (electron paramagnetic resonance). Regularly quality control testing of HDR treatment units is also a necessity to ensure accurate treatment dosimetry [76-79]. Several papers are available with alternative methods to undertake efficient and comprehensive testing, for example Yewondwossen et al. [80] have demonstrated the use of a 2D-array of ion chambers for brachytherapy dosimetric quality assurance and Devic et al. [81] have used radiochromic film to compare delivered doses with intended treatment plans.

Accurate determination of the HDR source strength is an obvious prerequisite for delivery of the intended dose distribution. However, even this step requires careful at- tention to avoid the potential for error, as reported by Dempsey [82] in which a well-type ionisation chamber calibration coefficient contained a $2.6 \%$ error from the calibration laboratory. There are several options to directly measure the absorbed dose to water in HDR brachytherapy to verify the source strength. Sarfehnia [83] has compared water calorimetry, ionization chamber and radiochromic film. Compared to water calorimetry, EBT-1 Gafchromic film, A1SL Exradin miniature Shonka thimble chamber and SI HDR 1000 Plus well-type chamber all agreed to within $0.8 \%$. The overall 1 sd uncertainty was $1.9 \%$ for water calorimetry, $1.4 \%$ for ionization chambers and $1.8 \%$ for Gafchromic film. Recent development of water calorimetrybased standards for absorbed dose to water in HDR brachytherapy have simplified the fundamental dosimetry formalism. Toni [84] and Quast et al. [85] provide reviews of the new brachytherapy dosimetry standards. Sarfehnia et al. 2010 [86] have reported on a water-calorimetry based system for Ir-192 dosimetry, demonstrating absorbed dose per air kerma strength agreement to within $0.8 \%$ with independent ionization chamber and EBT-1 Gafchromic film reference dosimetry. DeWerd et al. [11] has undertaken a full uncertainty analysis of brachytherapy dosimetry, considering each step in the dosimetry chain from standards laboratory to clinical brachytherapy department. A combined dosimetric uncertainty of $<5 \%(\mathrm{k}=1)$ is estimated.

Interdepartmental or mailed quality audits are also a valuable method of comparing dosimetric accuracy and contributing to safety, in specific brachytherapy clinics, as reviewed by Sibata and Gossman [87]. A brachytherapy audit was conducted in The Netherlands and Belgium, reported by Elfrink et al. [88], to assess accuracy of brachytherapy treatments, implant reconstruction and dose delivery. A cubic phantom containing positionally well-known metal spheres for reconstruction tests and another cylindrical phantom consisting of three catheters surrounding a centrally positioned NE2571 ionization chamber, were sent to 33 radiotherapy centres. An intended prescribed dose to the chamber which was compared to measurement, for HDR, PDR and LDR systems. The average dose difference was $+0.9 \%( \pm 1.3 \%, 1 \mathrm{sd})$ for 21 HDR units and the average applicator reconstruction accuracy was $-0.07 \mathrm{~mm}( \pm 0.4 \mathrm{~mm}$, $1 \mathrm{sd})$. A dosimetric intercomparison of brachytherapy treatment units in 9 radiotherapy centres in Ireland, Scotland and the North of England was reported by Heeney et al. [89]. A phantom was used to perform in-air measurements of source strength with a standard Farmer-type ionisation chamber. 5 HDR units were surveyed, with the difference between locally measured to audit measured source strengths of between $+1.5 \%$ to $-0.9 \%$. The quoted mean relative combined uncertainty was $1.3 \%$.

A quality control audit based on a mailed TLD system (LiF powder type DTL 937, Philitech) has been developed by the BRAPHYQS Physics Network and the EQUAL-ESTRO laboratory, as reported by Roue et al. [90]. The system measures the absorbed dose to water in the vicinity of an HDR or PDR source, at a uniform dose location at the centre of three source catheters housed in a purpose designed PMMA phantom. The aim is to compare the dose to water measured with the TLD and the dose calculated by the clin- 
ical TPS, with a total uncertainty budget of $3.3 \%$. At the time of publication (2007), 11 centres had undertaken the audit and one result was found outside of tolerance due partially to an incorrect calibration coefficient detected. The authors conclude that such external audit can be an efficient QC method complementary to internal quality control as it can reveal some errors which are not observable by other means.

An audit of source strength determination for HDR brachytherapy was conducted in 14 Swedish centres by Carlsson Tedgren et al. [91]. Values of reference air kerma rate (RAKR) were compared from the external audit, hospitals' measurements as well as vendors certificates, and all agreed within the uncertainty limits stated by the vendors. The use of robust well-type ionisation chambers was thought to contribute to the high levels of agreement. Austerlitz et al. [92] have proposed a Fricke dosimetry system for clinical HDR quality control audits via mail distribution. The prototype system consisted of a water phantom with the HDR source positioned at the centre of a spherical balloon filled with Fricke solution. The authors confirmed suitability of the system for assessment of source strength, decay and treatment planning parameters. Ochoa et al. [93] have reported on a phantom design for the verification of the dose calculated by the treatment planning system, using powder TLD capsules. An in-air measurement of source strength and in-water verification of the dose calculation algorithm are employed. The system was mailed to seven radiotherapy centres in Brazil, with comparisons between phantom measurement, well-type ionisation chamber and source specification certificate agreeing within $3 \%$. The authors conclude the phantom demonstrated its usefulness to verify the source strength and consistency of TPS to calculate the absorbed dose delivered to the patient, as part of a National Dosimetry Quality Assurance Program in Brazil. This work followed an earlier publication by de Almeida et al. [94] in which a pilot HDR dosimetry audit was conducted.

\section{Dose delivery verification}

Verification of dosimetric accuracy of the delivered treatment plan may ideally be assessed in vivo to record the actual dose delivered within the patient. However, there are a number of uncertainties with this approach, not least positional dependence of the dosimeter within the patient. An alternative, or perhaps additional, process is the prior verification of intended treatment delivery in a phantom. Qi et al. [95] have evaluated the use of a MOSFET dosimetry system within a custom made phantom to verify HDR brachytherapy plan delivery. Deviations between measured doses and treatment planning system calculated doses were all within 5\% for the plans considered.

The optimum detector to confirm in vivo dosimetric accuracy in HDR brachytherapy has not yet been established. A number of systems have been used and are being developed including passive integrating TLDs and active methods based on MOSFETS, diodes, optical fibre-coupled organic scintillators or doped silica glass. Lambert et al. [96] compared a scintillation detector (BrachyFOD) with a diamond detector, MOSFET and LiF TLD in the presence of an Ir-192 source. The diamond detector was found to be the most accurate, but had a large physical size, TLDs were not favoured due to inability of real-time readings and had depth dependent sensitivity, MOSFETs used in the study were subject to large errors at distances greater than $50 \mathrm{~mm}$ from the source. The authors concluded a scintillation detector could have the most favourable combination of characteristics for in vivo dosimetric verification in HDR brachytherapy.

The use of thermoluminescent dosimetry (TLD) is a traditional method for external beam verification and has also frequently been applied for in vivo use in HDR brachytherapy. Das et al. [97] reported on the use of LiF:Mg,Ti TLD rods of $1 \mathrm{~mm}$ diameter, with up to 11 detectors positioned every $16 \mathrm{~mm}$ separated by radio-opaque markers, in catheters within the urethra and rectum of prostate cancer patients. Hsu et al. [98] compared the use of a radiophotoluminescent glass dosimeter (RPLGD) to a traditional thermoluminescent dosimeter for the dose verification of prostate HDR brachytherapy. The authors concluded the RPLGD demonstrated good repeatability, stability and small effective size, and showed promise as a reliable dosimeter for in vivo dosimetry with clinically acceptable accuracy.

The use of remote optical fibre dosimetry in brachytherapy [99] has received some attention in brachytherapy. Andersen et al. [100] characterized a fibre-coupled $\mathrm{Al}_{2} \mathrm{O}_{3}: \mathrm{C}$ luminescence dosimetry system for online in vivo dose measurement with an Ir-192 brachytherapy source, to facilitate prevention and identification of dose delivery errors. The system uses both radioluminescence (RL) and optically stimulated luminescence (OSL) from aluminium oxide crystals attached to optical fibre cables. The authors found the system was adequate for in vivo brachytherapy dosimetry.

A fibre optic dosimetry system was also developed by Suchowerska et al. [101] to measure urethral and rectal wall doses in HDR prostate patients. In the study of 25 patients, the dosimetry system indicated a maximum departure of $9 \%$ from the calculated dose in the urethra. The authors indicate knowledge of the actual dose delivered could be used to correlate to toxicity, or as a warning of potential over or under dose. Kertzscher et al. [102] have also investigated the use of a real-time fibre-coupled carbon doped aluminium oxide $\left(\mathrm{Al}_{2} \mathrm{O}_{3}: \mathrm{C}\right)$ crystal dosimeter for in vivo dosimetry detection of imposed treatment errors in phantom studies, for gynaecological and prostate treatment plans. Interchanged guide tubes and 2 to $20 \mathrm{~mm}$ source displacements were monitored with the detector in the tumour region. It was found applicator displacements greater or equal to $5 \mathrm{~mm}$ and interchanged guide tube errors could be detected by real-time dosimetry. Therriault-Proulx et al. [103] have also used a plastic scintillation detector (PSD) coupled to a plastic optical fibre to perform in-phantom dosimetry during Ir-192 HDR brachytherapy treatments. Prostate plan HDR treatments were delivered to the PSD in water, comparing the measured values to treatment planning system calculations. The PSD is designed for verification of dose in rectum and urethra. An intentional $5 \mathrm{~mm}$ displacement of the source was detected by the PSD in over $78 \%$ of delivered plans. The study also demonstrated the need to implement a stem effect removal technique for the PSD signal, which the author had previously reported in more detail [104]. The authors state the system has the potential for various 
online verifications of treatment delivery quality in HDR brachytherapy.

There have been a number of publications assessing the possibility of using metal oxide semiconductor field effect transistor detectors (MOSFETs) as in vivo dosimeters [105]. Haughey et al. [106] assessed the suitability of linear array MOSFETs as in vivo dosimeters to measure rectal dose in HDR cervix brachytherapy treatments. MOSFET dosimeters are attractive due to their small sensitive volume and direct readout. CT scans were obtained with the MOSFETs inserted into the patients' rectum and measured rectal doses during treatment were compared with point doses calculated by the treatment planning system (TPS). For prostate patients, only $33 \%$ of measured doses agreed with the TPS within $10 \%$ and for cervix, $42 \%$ agreed within $10 \%$. Due to the many uncertainties associated with MOSFETs including calibration drift, angular dependence and the inability to know their exact position at the time of treatment, Haughey et al. considered them to be unsuitable for rectal in vivo dosimetry in HDR brachytherapy. Reniers et al. [107] also evaluated the performance of MOSFET detectors for in vivo dosimetry to verify correct dose delivery in gynaecological HDR brachytherapy. It was acknowledged that information on dosimeter position with respect to the source is paramount to the interpretation of in vivo measurements due to high dose gradients that are present.

The RADPOS system (Best Medical, Canada) consists of a MOSFET dosimeter physically coupled to a position-sensing probe, which deduces its 3D position in static magnetic fields generated by a transmitter, shown to be accurate within 0.5 to $1.0 \mathrm{~mm}$. MC code was used to derive the photon energy spectrum at the known distance from the source, to interpret the MOSFET reading. The authors concluded the system showed promise as an in vivo dosimetry systemfor HDR brachytherapy. Able et al. [18] have also used a MOSFET dosimetry system as the detectors in a prostate phantom for HDR treatment. The purpose of the work was to assess the application of statistical process control (SPC) as a quality control method to ensure the treatment was delivered with minimal variation. CT guidance was used for positioning of detectors, exposed to treatments delivered accurately and with induced errors. SPC methodology was shown to be a valuable approach with further work required on determining the most effective positioning of the detectors. Anton et al. (2009) [109] have conducted a prostate phantom study to demonstrate the potential of alanine/ESR as an in vivo dosimeter with an Ir-192 source. The uncertainty of measurement within a Foley catheter for dose measurements within the urethra was estimated to be $3.6 \%$, concluding this to be suitable for clinical in vivo measurements. Holly et al. [110] used a conebeam CT imaging technique to verify the positioning of HDR applicators prior to treatment delivery, in prostate brachytherapy. The mean internal displacement of the catheters was $11 \mathrm{~mm}$, resulting in a decrease in mean volume receiving $100 \%$ of prescription dose (V100) from planned $97.6 \%$ to $77.3 \%(p<0.001)$. Each $1 \mathrm{~cm}$ of catheter displacement resulted in a decrease of $20 \%$ in V100 and a reduction in the mean dose to $90 \%$ of the prostate (D90) of $36 \%$. The study showed internal displacement of HDR catheters for prostate treatment is common, but can be partly corrected if detected. These results are similar to the work of Foster et al. [111] who also evaluated the impact of interfraction catheter movement on dosimetry in prostate HDR brachytherapy using CT. A mean displacement of $5.1 \mathrm{~mm}$ was recorded, resulting in prostate V100 reduction from $93.8 \%$ to $76.2 \%$, considered significant by the authors and required re-optimisation before the second treatment. Meiln Bermejo et al. [112] have developed the use of Gafchromic EBT2 film to confirm the correct dose delivery to the surface of the skin in skin cancer treatments. A significant challenge in dosimetry is the surface-dose determination because of its experimental complexity. Measurements with EBT2 were shown to be a suitable method for QA purposes in HDR brachytherapy. Hassouna et al. [113] have shown that in vivo dosimetry using diodes can provide appropriate quality control of HDR brachytherapy treatments in cervix cancer. Following calibration and a phantom study to estimate the reproducibility $(0.5 \%)$ and uncertainty $(1.9 \%)$ of the in vivo dosimetry system, a prospective study using the detectors in 32 intracavitary brachytherapy implants was conducted. $48 \%$ of rectal diode measurements and $46 \%$ of bladder diode measurements were more than $10 \%$ different from TPS expected dose. The cause was likely difficulties in position verification in high dose gradients.

The method of interpretation of in vivo dosimetry results must be considered alongside the development of dosimetry systems themselves, in order to interpret the accuracy of treatment delivery and take clinical decision based on measurement results. Toye et al. [114] have developed an in vivo dosimetry based investigative action level relevant for a corrective protocol for HDR brachytherapy prostate boost treatment. This was based on urethra and rectum thermoluminescent (TLD) dosimetry in 56 patients. An initial investigative level of $20 \%$ difference between measurement and treatment planning system was established, corresponding to $1 / 3$ of patients.

\section{Discussion}

A review of recent published work concerned with improving or verifying the dosimetric accuracy of HDR brachytherapy treatments has been presented. A wide range of tools are currently being employed to achieve this aim, with no consensus on the optimum method for each element of the process, except in the determination of HDR source dose rate distribution where MC methods prevail. Even for these calculations, the majority of investigators use an additional experimental technique for validation of calculation results, which may include point dose TLDs, 2D radiochromic film and 3D gel/plastic dosimeters. The benefits of particular detector systems are related not only to their physical properties, such as size, dose resolution, response, uncertainty, but also to practical usability in brachytherapy. For verification of MC-derived dose distributions around individual source models, a full-3D gel dosimeter may be desirable, but is impractical for routine quality control checks where radiochromic film or ionisation chambers provide more immediate results. 
The lack of correction for tissue or applicator inhomogeneities and patient-specific scattering conditions in brachytherapy treatment planning systems is well known, but the effect on dose distributions and clinical implications has less agreement; with estimates ranging from 3 to $25 \%$ in different conditions. It is likely that model-based advanced planning systems will become more widespread in the near future to reduce this uncertainty. There is also scope to improve the use of treatment planning systems to achieve the desired dose distribution, development of new applicators and a gradual move away from traditional brachytherapy loading systems. Equipment performance should not be forgotten as an essential step in ensuring dosimetric accuracy, both in initial comprehensive commissioning and regular quality control testing. There appears to be a lack of interdepartmental audits, clinical trials quality assurance, or routine external audit testing for HDR brachytherapy in the published literature. The majority of publications are related to Ir-192, this being the most common isotope in clinical use, with a lack of work for newer HDR sources such as Co-60. In vivo verification of dose delivery has received much attention, with investigators using a number of tools including TLD, MOSFET, semiconductor diodes, remote optical fibre dosimetry, alanine and radiochromic film. However, accurately verifying the precise location of the detectors in the steep dose gradients involved in brachytherapy treatment has restricted the quality that has been achieved with in vivo measurements and relatively high uncertainties remain. Attention may be better directed towards a combination of verification of the equipment performance during treatment with improved image-guidance to ensure correct relative locations of applicators and internal structures. Imaging verification of brachytherapy delivery requires further development. There has been some work confirming applicator position, but only limited studies on in vivo assessment of the correlation of internal organ motion with respect to applicators, namely in prostate HDR. The correct identification, reconstruction and position of applicators are critical to the accuracy of brachytherapy. The extensive development and adoption of advanced image-guidance for external beam radiotherapy may be repeated for brachytherapy in the near future.

As the complexity of brachytherapy delivery increases, there is also scope to develop pre-treatment delivery checks, as is common for external beam intensity-modulated radiotherapy. A required nominal overall accuracy for brachytherapy dose delivery of $3 \%$ is sensible [4]. However, in assessing the accuracy of the delivered dose distribution compared to the plan, there is a lack of an accepted definition of quality. The application of gamma-type assessments of intended compared to delivered dose distributions would be of value for brachytherapy.

It is difficult to specify and quantify the current achievable accuracy in HDR brachytherapy treatments. The accuracy of physical dose delivery, including source strength calibration, is expected to be better than $3 \%$. Treatment planning system accuracy, using TG-43 approach, is generally within $2 \%$ of measured or MC calculated doses, although uncertainties increase in the presence of inhomogeneities. Other determinants of accuracy such as variations in treat- ment equipment performance have not been comprehensively evaluated within HDR brachytherapy treatments. The uncertainties of greatest magnitude in brachytherapy treatment are currently likely to be associated with 'clini-

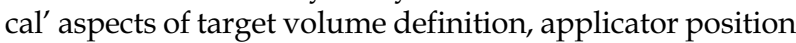
and movement and the effect of internal organ movement.

In terms of overall dosimetric accuracy of brachytherapy treatments, it may be useful to consider quality system approaches, to target research work in those areas with the largest current uncertainty or indeed where there is the greatest potential for treatment error. Swamidas et al. [115] have evaluated the safety of intracavitary brachytherapy using failure mode and effects analysis (FMEA) according to a method being used by the American Association of Physicists in Medicine (AAPM) Task Group No 100, assigning risk probability to all process elements. Similarly, Thomadsen et al. [12] have applied formal risk analysis techniques to dosimetric accuracy of brachytherapy.

\section{Conclusions}

Assuring the dosimetric accuracy of HDR brachytherapy, reducing uncertainties and improving dose delivery optimisation, are all areas where significant development and research work is being undertaken, with many varied tools being employed for both calculation and experimental investigation. In 'source dosimetry', MC techniques are well established and TLD, radiochromic film, as well as gel dosimeters have all been used to provide verification of calculations. However, there is often a lack of consideration of the size and shape of phantoms and scatter conditions used in this work. In 'treatment planning', new model-based dose calculation algorithms will revolutionise the uncertainty associated with current TG- 43 based techniques in the presence of inhomogeneities and differing scatter conditions. Further work is required to quantify the extent of dose distribution differences that will occur in typical treatment situations with the new TPS algorithms and indeed the magnitude of current TG-43 uncertainties. In 'equipment performance', guidance exists on local quality control practices, but there is a lack of quality audits when compared to similar activity in external beam radiotherapy. Source strength measurements are also the subject of much current research and development attention. In 'dose delivery verification', techniques for reliable in vivo dose measurement have not been practically realised and therefore are not in widespread use. Further development is required in combination with improved image-guidance of brachytherapy. The development of routine methods for the accurate determination of applicator location, target and organs at risk is required.

\section{References}

1. ICRU Report 24. Determination of Absorbed Dose in a Patient Irradiated by Beams of X or Gamma Rays in Radiotherapy Procedures. International Commission on Radiation Units and Measurement, 1976.

2. Brahme A. Dosimetric precision requirements in radiation therapy. Acta Radiol Oncol 1984; 23: 379-391.

3. Mijnheer BJ, Battermann JJ, Wambersie A. What degree of accuracy is required and can be achieved in photon and neutron therapy? Radiother Oncol 1987; 8: 237-252. 
4. Van Dyk J, Barnett RB, Cygler JE et al. Commissioning and quality assurance of treatment planning computers. Int J Radiat Oncol Biol Phys 1993; 20: 261-273.

5. IAEA Safety Report Series No. 17. Lessons learned from accidental exposures in radiotherapy. International Atomic Energy Agency, Vienna 2000.

6. ICRP Publication 86. Prevention of accidents to patients undergoing radiation therapy. International Commission on Radiological Protection, 2000.

7. ICRP Publication 97. Prevention of high-dose-rate brachytherapy accidents. International Commission on Radiological Protection, 2005.

8. Thomadsen BR, Williamson JF, Rivard MJ et al. Anniversary paper: past and current issues, and trends in brachytherapy physics. Med Phys 2008; 35: 4708-4723.

9. Ibbott G, Ma CM, Rogers DWO et al. Anniversary Paper: Fifty years of AAPM involvement in radiation dosiemtry. Med Phys 2008; 35: 1418-1427.

10. Ibbott GS, Attix FH, Slowey TW et al. Uncertainty of calibrations at the accredited dosimetry calibration laboratories. Med Phys 1997; 24: 1249-1254.

11. DeWerd LA, Ibbott GS, Meigooni AS et al. A dosimetric uncertainty analysis for photon-emitting brachytherapy sources: report of AAPM Task Group No. 138 and GEC-ESTRO. Med Phys 2011; 38: 782-801.

12. Thomadsen B, Lin SW, Laemmrich P et al. Analysis of treatment delivery errors in brachytherapy using formal risk analysis techniques. Int J Radiat Oncol Biol Phys 2003; 57: 1492-1508.

13. Hoskin PJ, Bowne, PJ, Ostler P et al. High dose rate afterloading brachytherapy for prostate cancer: catheter and gland movement between fractions. Radiother Oncol 2003; 68: 285-288.

14. Whitaker M, Hruby G, Lovett A et al. Prostate HDR brachytherapy catheter displacement between planning and treatment delivery. Radiother Oncol 2011; 101: 490-494.

15. D'Souza D, Baldassarre F, Morton G et al. Imaging technologies for high dose rate brachytherapy for cervical cancer: a systematic review. Clin Oncol 2011; 23: 460-475.

16. Williamson JF, Dunscombe PB, Sharpe MB et al. Quality assurance needs for modern image-based radiotherapy: recommendations from 2007 interorganiational symposium on "quality assurance of radiation therapy: challenges of advanced technology". Int J Radiat Oncol Biol Phys 2008; 71: S2-12.

17. Karaiskos P, Angelopoulos A, Sakelliou L et al. Monte Carlo and TLD dosimetry of an Ir-192 high dose-rate brachytherapy source. Med Phys 1998; 25: 1975-1984.

18. Kirov AS, Williamson JF, Meigooni AS et al. TLD, diode and Monte Carlo dosimetry of an Ir-192 source for high dose-rate brachytherapy. Phys Med Biol 1995; 40: 2015-2036.

19. Muller-Runkel R, Cho SH. Anisotropy measurements of a high dose rate Ir-192 source in air and in polystyrene. Med Phys 1994; 21: 1131-1134.

20. Zhang M, Zhang L, Yuan S et al. A study of experimental measurements of dosimetric parameters in HDR Ir-192 source. Med Dosim 2010; 35: 250-254

21. Mishra V, Waterman FM, Suntharalingam N. Anisotropy of an 192iridium high dose rate source measured with a miniature ionization chamber. Med Phys 1997; 24: 751-755.

22. Zilio VO, Joneja OP, Popowski Y et al. Absolute depth-doserate measurements for an Ir-192 HDR brachytherapy source in water using MOSFET detectors. Med Phys 2006; 33: 15321539.

23. Toye WC, Das KR, Todd SP et al. An experimental MOSFET approach to characterize (192)Ir HDR source anisotropy. Phys Med Biol 2007; 52: 5329-5339.

24. Aldelaijan S, Mohammed H, Tomic N et al. Radiochromic film dosimetry of HDR (192)Ir source radiation fields. Med Phys 2011; 38: 6074-6083.
25. Butson MJ, Yu PKN, Cheung T et al. Radiochromic film for medical radiation dosimetry. Mat Sci Eng R 2003; 41: 61-120.

26. Sharma SD, Bianchi C, Conte L et al. Radiochromic film measurement of anisotropy function for high-dose-rate Ir-192 brachytherapy source. Phys Med Biol 2004; 49: 4065-4072.

27. Sureka CS, Sunny CS, Subbaiah KV et al. Dose distribution for endovascular brachytherapy using Ir-192 sources: comparison of Monte Carlo calculations with radiochromic film measurements. Phys Med Biol 2007; 52: 525-537.

28. Chiu-Tsao ST, Duckworth TL, Patel NS et al. Verification of Ir192 near source dosimetry using GAFCHROMIC film. Med Phys 2004; 31: 201-207.

29. Baras P, Seimenis I, Kipouros P et al. Polymer gel dosimetry using a three-dimensional MRI acquisition technique. Med Phys 2002; 29: 2506-2516.

30. Williamson JF, Li Z. Monte Carlo aided dosimetry of the microSelectron pulsed and high dose-rate Ir-192 sources. Med Phys 1995; 22: 809-819.

31. Daskalov GM, Loffler E, Williamson JF. Monte Carlo-aided dosimetry of a new high dose-rate brachytherapy source. Med Phys 1998; 25: 2200-2208.

32. Papagiannis P, Angelopoulos A, Pantelis E et al. Dosimetry comparison of Ir-192 sources. Med Phys 2002; 29: 2239-2246.

33. Sureka CS, Aruna P, Ganesan S et al. Computation of relative dose distribution and effective transmission around a shielded vaginal cylinder with Ir-192 HDR source using MCNP4B. Med Phys 2006; 33: 1552-1561.

34. Granero D, Pérez-Calatayud J, Ballester F. Monte Carlo calculation of the TG-43 dosimetric parameters of a new BEBIG Ir192 HDR source. Radiother Oncol 2005; 76: 79-85.

35. Papagiannis P, Angelopoulos A, Pantelis E et al. Monte Carlo dosimetry of 60Co HDR brachytherapy sources. Med Phys 2003; 30: 712-721.

36. Lux I, Koblinger L. Monte Carlo Particle Transport Methods. Neutron and Photon Calculations. CRC Press, Boca Raton 1991.

37. Nath R, Anderson LL, Luxton G et al. Dosimetry of interstitial brachytherapy sources: Recommendations of the AAPM Radiation Therapy Committee Task Group No. 43. Med Phys 1995; 22: 209-234

38. Rivard MJ, Coursey BM, DeWerd LA et al. Update of AAPM Task Group No. 43 Report. A revised AAPM protocol for brachytherapy dose calculations. Med Phys 2004; 31: 633-674.

39. Williamson J, Coursey BM, DeWerd LA et al. Dosimetric prerequisites for routine clinical use of new low energy photon interstitial brachytherapy sources. Med Phys 1998; 25: 22692270.

40. Daskalov GM, Loffler E, Williamson JF. Monte Carlo-aided dosimetry of a new high dose-rate brachytherapy source. Med Phys 1998; 25: 2200-2208.

41. Chandola RM, Tiwari S, Kowar MK et al. Monte Carlo and experimental dosimetric study of the mHDR-v2 brachytherapy source. J Can Res Ther 2010; 6: 421-426.

42. Lliso F, Granero D, Pérez-Calatayud J et al. Dosimetric evaluation of internal shielding in a high dose rate skin applicator. J Contemp Brachyther 2011; 3: 32-35.

43. Uniyal SC, Sharma SD, Naithani UC. A dosimetry method in the transverse plane of HDR Ir-192 brachytherapy source using gafchromic EBT2 film. Phys Med 2012; 28: 129-133; DOI: 10.1016/j.ejmp.2011.03.005.

44. Deene YD, Reynaert N, Wagter CD. On the accuracy of monomer/polymer gel dosimetry in the proximity of a high-doserate Ir-192 source. Phys Med Biol 2001; 46: 2801-2825.

45. Guo PY, Adamovics JA, Oldham M. Characterization of a new radiochromic three-dimensional dosimeter. Med Phys 2006; 33: 1338-1345. 
46. Wai P, Adamovics J, Krstajic N et al. Dosimetry of the microSelectron-HDR Ir-192 source using PRESAGETM and optical CT. Appl Radiat Isotopes 2009; 67: 419-422.

47. Guo P, Adamovics J, Oldham M. A practical three-dimensional dosimetry system for radiation therapy. Med Phys 2006; 33: 3962-3972.

48. Mostaar A, Hashemi B, Zahmatkesh MH et al. A basic dosimetric study of PRESAGE: the effect of different amounts of fabricating components on the sensitivity and stability of the dosimeter. Phys Med Biol 2010; 55: 903-912.

49. Gorjiara T, Hill R, Kuncic Z et al. Investigation of radiological properties and water equivalency of PRESAGE ${ }^{\circledR}$ dosimeters. Med Phys 2011; 38: 2265-2274.

50. Carrara M, Gambarlnl G, Scotti A et al. Fricke gel dosimetry for the measurement of the anisotropy function of an Ir-192 HDR brachytherapy source. Radiother Oncol 2010; 96: S516S517.

51. Antonovic L, Gustafsson H, Carlsson GA et al. Evaluation of a lithium formate EPR dosimetry system for dose measurements around 192Ir brachytherapy sources. Med Phys 2009; 36: 2236-2247.

52. Issa F, Rahman ATA, Hugtenburg RP et al. Establishment of Ge-doped optical fibres as thermoluminescence dosimeters for brachytherapy. Appl Radiat Isotopes 2012; 70: 1158-1161; DOI: 10.1016/j.apradiso.2011.12.027.

53. Granero D, Pérez-Calatayud J, Pujades-Claumarchirant MC et al. Equivalent phantom sizes and shapes for brachytherapy dosimetric studies of 192Ir and 137Cs. Med Phys 2008; 35: 4872-4877.

54. Rivard MJ, Venselaar JL, Beaulieu L. The evolution of brachytherapy treatment planning. Med Phys 2009; 36: 2136-2153.

55. Rivard MJ, Beaulieu L, Mourtada F. Enhancements to commissioning techniques and quality assurance of brachytherapy treatment planning systems that use model-based dose calculation algorithms. Med Phys 2010; 37: 2645-2658.

56. AAPM Task Group No. 186 Model-based Dose Calculation Techniques in Brachytherapy: Status and Clinical Requirements for Implementation Beyond AAPM TG-43. The American Association of Physicists in Medicine; http://www.aapm. org/org/structure/default.asp?committee_code=TG186 (accessed 24/04/2012).

57. Chavez-Aguilera N, Torres-Garcia E, Mitsoura E. Effect of chemical composition and density of the pelvic structure in intracavitary brachytherapy dosimetry. Rad Phys Chem 2011; 80: 349-353.

58. Parsai EI, Shang Z, Feldmeier JJ. A quantitative three-dimensional dose attenuation analysis around fletcher-Suit-Delclos due to stainless steel tube for high-dose-rate brachytherapy by Monte Carlo calculations. Brachytherapy 2009; 8: 318-323.

59. Uniyal SC, Naithani UC, Sharma SD et al. Radiochromic film dosimetry of rectal inhomogeneity and applicator attenuation in high dose rate brachytherapy of uterine cervix. J Appl Clin Med Phys 2012; 13: 3654.

60. Desbiens M, D'Amours M, Afsharpour H et al. Monte Carlo dosimetry of high dose rate gynaecologic interstitial brachytherapy. Radiother Oncol 2011; 99: S59.

61. Lakshminarayanan T, Subbaiah KV, Thayalan K et al. Suitability of point kernel dose calculation techniques in brachytherapy treatment planning. J Med Phys 2010; 35: 88-99.

62. DeMarco J, Park S, Kamrava M et al. A monte carlo analysis of HDR absorbed dose calculations using surface applicators. Int J Radiat Oncol Biol Phys 2011; 81: S895.

63. Zourari K, Pantelis E, Moutsatsos A et al. A virtual phantom/ dicom protocol-based QA procedure for contemporary HDR 192Ir brachytherapy TPS. Radiother Oncol 2011; 99: S404-S405.
64. Zourari K, Pantelis E, Moutsatsos A et al. Dosimetric accuracy of a deterministic radiation transport based 192Ir brachytherapy treatment planning system. Part 1: Single sources and bounded homogeneous geometries. Med Phys 2010; 37: 649-661.

65. Petrokokkinos L, Zourari K, Pantelis E et al. Dosimetric accuracy of a deterministic radiation transport based 192Ir brachytherapy treatment planning system. Part II: Monte Carlo and experimental verification of a multiple source dwell position plan employing a shielded applicator. Med Phys 2011; 38: 1981-1992.

66. Citrin D, Ning H, Guion P et al. Inverse treatment planning based on MRI for HDR prostate brachytherapy. Int J Radiat Oncol Biol Phys 2005; 61: 1267-1275.

67. Brooks S, Bownes P, Lowe G et al. Cervical brachytherapy utilizing ring applicator: Comparison of standard and conformal loading. Int J Radiat Oncol Biol Phys 2005; 63: 934-939.

68. Siauw T, Cunha A, Atamturk A et al. IPIP: A new approach to inverse planning for HDR brachytherapy by directly optimizing dosimetric indices. Med Phys 2011; 38: 4045-4051.

69. Kumar R, Sharma S, Vijaykumar C et al. A dose verification method for high-dose-rate brachytherapy treatment plans. J Can Res Ther 2008; 4: 173-177.

70. Astrahan MA. HDR quality assurance methods for personal digital assistants. Med Dosim 2004; 29: 166-172.

71. Carmona V, Pérez-Calatayud J, Lliso F et al. A program for the independent verification of brachytherapy planning system calculations. J Contemp Brachyther 2010; 2: 129-133.

72. Cormack RA. Quality assurance issues for computed tomography-, ultrasound-, and magnetic resonance imaging-guided brachytherapy. Int J Radiat Oncol Biol Phys 2008; 71: S136S141.

73. Palmer A, Mzenda B. Performance assessment of the BEBIG MultiSource high dose rate brachytherapy treatment unit. Phys Med Biol 2009; 54: 7417-7434.

74. Sahoo N. Measurement of transit time of a remote after-loading high dose rate brachytherapy source. Med Phys 2001; 28: 1786-1790.

75. Calcina CS, de Almeida A, Rocha JR et al. Ir-192 HDR transit dose and radial dose function determination using alanine/EPR dosimetry. Phys Med Biol 2005; 50: 1109-1117.

76. Kutcher GJ, Coia L, Gillin M et al. Comprehensive QA for radiation oncology. Report of the AAPM Radiation Therapy Committee Task Group No. 40. Med Phys 1994; 21: 581-618.

77. Nath R, Anderson L, Meli JA et al. Code of practice for brachytherapy physics: Report of the AAPM Radiation Therapy Committee Task Group No. 56. Med Phys 1997; 24: 1557-1598.

78. IPEM Report 81. Physics aspects of quality control in radiotherapy. Institute of Physics and Engineering in Medicine, New York 1999.

79. Bidmead M, Briot E, Burger J et al. A practical guide to quality control of brachytherapy equipment. ESTRO Booklet No. 8 . European Society for Therapeutic Radiology and Oncology, Brussels 2004

80. Yewondwossen M. Characterization and use of a 2D-array of ion chambers for brachytherapy dosimetric quality assurance. Med Dosim 2011 [Epub ahead of print]; PMID: 22189031.

81. Devic S, Tomic N, DeBlois F. Brachytherapy TPS QA Using EBT Model GafChromic Film. Med Phys 2009; 36: 2528-2528.

82. Dempsey C. Lessons learned from a HDR brachytherapy well ionisation chamber calibration error. Australas Phys Eng Sci Med 2011; 34: 529-533.

83. Sarfehnia A, Kawrakow I, Seuntjens J. Direct measurement of absorbed dose to water in HDR 192Ir brachytherapy: water calorimetry, ionization chamber, Gafchromic film, and TG-43. Med Phys 2010; 37: 1924-1932. 
84. Toni MP. New brachytherapy standards paradigm shift. In: Proceedings of an International Symposium on Standards, Applications and Quality Assurance in Medical Radiation Dosimetry (IDOS, 2010). International Atomic Energy Agency, Vienna 2011; 1: 393-406.

85. Quast U, Kaulich TW, Ahnesjö A et al. From reference airkerma-rate to nominal absorbed dose-rate to water - Paradigm shift in photon brachytherapy: ISO new work item proposal. In: Proceedings of an International Symposium on Standards, Applications and Quality Assurance in Medical Radiation Dosimetry (IDOS, 2010). International Atomic Energy Agency, Vienna 2011; 1: 407-416.

86. Sarfehnia A, Seuntjens J. Development of a water calorimetry-based standard for absorbed dose to water in HDR 192Ir brachytherapy. Med Phys 2010; 37: 1914-1923.

87. Sibata CH, Gossman MS. Role of quality audits: view from North America. In: Quality and safety in radiotherapy (Imaging in medical diagnosis and therapy). Pawlicki T, Dunscombe P, Mundt AJ, Scaillet P (eds.). Taylor \& Francis, Boca Raton 2010, pp. 161-165.

88. Elfrink RJM, Kolkman-Deurloo I-KK, van Kleffens HJ et al. Determination of the accuracy of implant reconstruction and dose delivery in brachytherapy in The Netherlands and Belgium. Radiother Oncol 2001; 59: 297-306.

89. Heeney C, McClean B, Kelly C. A dosimetric intercomparison of brachytherapy facilities in Ireland, Scotland and the North of England. Radiother Oncol 2005; 74: 149-156.

90. Roué A, Venselaar JL, Ferreira IH et al. Development of a TLD mailed system for remote dosimetry audit for (192)Ir HDR and PDR sources. Radiother Oncol 2007; 83: 86-93.

91. Carlsson Tedgren A, Grindborg JE. Audit on source strength determination for HDR and PDR Ir-192 brachytherapy in Sweden. Radiother Oncol 2008; 86: 126-130.

92. Austerlitz C, Mota H, Almeida CE et al. Quality assurance of HDR 192Ir sources using a Fricke dosimeter. Med Phys 2007; 34: 1348-53.

93. Ochoa R, Gomes F, Ferreira IH et al. Design of a phantom for the quality control of high dose rate 192-Ir source in brachytherapy. Radiother Oncol 2007; 82: 222-228.

94. deAlmeida CE, Pereira AJ, Marechal MH et al. Intercomparison of calibration procedures for 192Ir HDR sources in Brazil. Phys Med Biol 1999; 44: N31-N38.

95. Qi ZY, Deng XW, Husang SM et al. Verification of the plan dosimetry for high dose rate brachytherapy using metaloxide-semiconductor field effect transistor detectors. Med Phys 2007; 34: 2007-2013.

96. Lambert J, Nakano T, Law S et al. In vivo dosimeters for HDR brachytherapy: a comparison of a diamond detector, MOSFET, TLD, and scintillation detector. Med Phys 2007; 34: 1759-1765.

97. Das R, Toye W, Kron T et al. Thermoluminescence dosimetry for in-vivo verification of high dose rate brachytherapy for prostate cancer. Australas Phys Eng Sci Med 2007; 30: 178-184.

98. Hsu SM, Yeh CY, Yeh TC et al. Clinical application of radiophotoluminescent glass dosimeter for dose verification of prostate HDR procedure. Med Phys 2008; 35: 5558-5564.

99. Huston AL, Justus BL, Falkenstein PL et al. Remote optical fiber dosimetry. Nucl Instr Meth Phys Res B 2001; 184: 55-67.

100. Anderson CE, Nielson SK, Greilich S et al. Characterization of a fiber-coupled Al2O3:C luminescence dosimetry system for online in vivo dose verification during 192Ir brachytherapy. Med Phys 2009; 36: 708-718.

101. Suchowerska N, Abolfathi P, Cartwright L et al. Clinical use of a fibre optic dosimeter array in brachytherapy: urethral dosimetry and rectal wall dose mapping. Radiother Oncol 2010; 96: S209.
102. Kertzscher G, Andersen CE, Siebert FA et al. Identifying afterloading PDR and HDR brachytherapy errors using real-time fiber-coupled $\mathrm{Al}(2) \mathrm{O}(3): \mathrm{C}$ dosimetry and a novel statistical error decision criterion. Radiother Oncol 2011; 100: 456-462.

103. Therriault-Proulx F, Briere TM, Mourtada F et al. A phantom study of an in vivo dosimetry system using plastic scintillation detectors for real-time verification of 192Ir HDR brachytherapy. Med Phys 2011; 38: 2542-2551.

104. Terriault-Proulx F, Beddar S, Briere TM et al. Technical note: removing the stem effect when performing Ir-192 HDR brachytherapy in vivo dosimetry using plastic scintillation detectors: a relevant and necessary step. Med Phys 2011; 38: 2176-2179.

105. Kinhikar RA, Sharma PK, Tambe CM et al. Dosimetric evaluation of a new OneDose MOSFET for Ir-192 energy. Phys Med Biol 2006; 51: 1261-1268.

106. Haughey A, Coalter G, Mugabe K. Evaluation of linear array MOSFET detectors for in vivo dosimetry to measure rectal dose in HDR brachytherapy. Australas Phys Eng Sci Med 2011; 34: 361-366.

107. Reniers B, Landry G, Eichneer R et al. In vivo dosimetry for gynaecological brachytherapy based on a novel radpos system. Radiother Oncol 2011; 99: S118-S119.

108. Able CM, Bright M, Frizzell B et al. Quality control of highdose-rate brachytherapy: Treatment delivery analysis using statistical process control. Brachytherapy 2010; 9: S64-65.

109. Anton M, Wagner D, Selbach HJ et al. In vivo dosimetry in the urethra using alanine/ESR during (192)Ir HDR brachytherapy of prostate cancer - a phantom study. Phys Med Biol 2009; 54: 2915-2931.

110. Holly R, Morton GC, Sankreacha R et al. Use of cone-beam imaging to correct for catheter displacement in high dose-rate prostate brachytherapy. Brachytherapy 2011; 10: 299-305.

111. Foster W, Cunha JAM, Hsu IC et al. Dosimetric impact of interfraction catheter movement in high-dose rate prostate brachytherapy. Int J Radiat Oncol Biol Phys 2011; 80: 85-90.

112. Meilán Bermejo E, Del Moral F, Pereira L et al. Calibration and use of Gafchromic EBT2 films for QA in HDR brachytherapy. Radiother Oncol 2011; 99: S405.

113. Hassouna A, Bahadur Y, Constantinescu C et al. Role of invivo diodes dosimetry for critical organ dose evaluation in HDR brachytherapy of cervix cancer. Radiother Oncol 2011; 99: S401-S402.

114. Toye W, Das R, Kron T et al. An in vivo investigative protocol for HDR prostate brachytherapy using urethral and rectal thermoluminescence dosimetry. Radiother Oncol 2009; 91: 243-248.

115. Swamidas JV, Sharma S, Mahantshetty UM et al. Risk assessment in intracavitary brachytherapy based on failure mode and effective analysis. Brachytherapy 2010; 9: S48. 